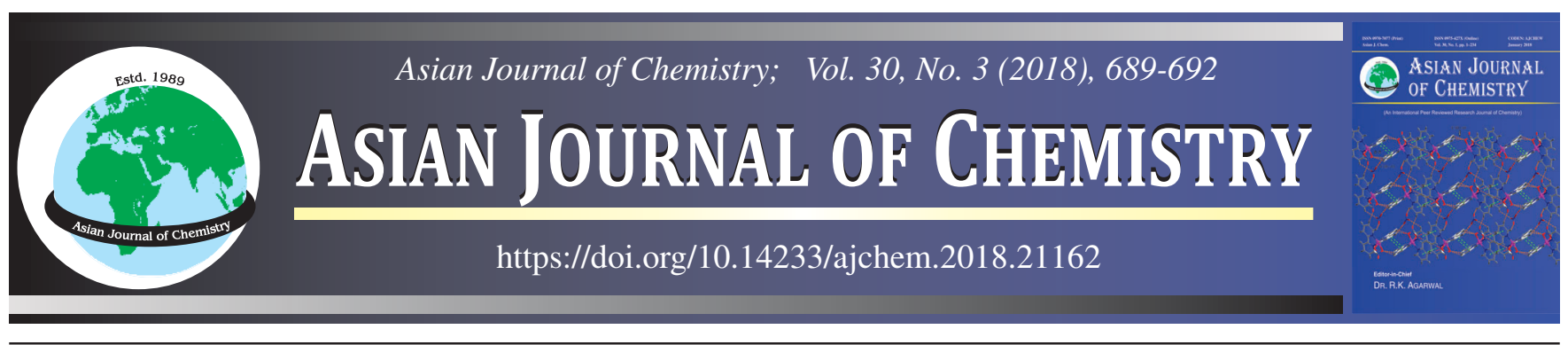

\title{
Empirical Two Step Approximation for Characterizing Thermal Properties of Ultrathin Graphite Sheet
}

\author{
Ju Yong $\mathrm{CHO}^{1}$, Seung Hoon $\mathrm{LeE}^{2}$ and Won Kweon JANG ${ }^{1, *}$
}

${ }^{1}$ Department of Electronic Engineering, Hanseo University, Seosan-si, Chungcheongnam-do 31962, Republic of Korea

${ }^{2}$ Department of Satellite Payload Technology, Korea Aerospace Research Institute, Daejeon 34133, Republic of Korea

*Corresponding author: Fax: +82 41 6883448; Tel: +82 41 6601324; E-mail: jwk@ hanseo.ac.kr; wonkjang@naver.com

Received: 2 December 2017;

Accepted: 20 December 2017;

Published online: 31 January 2018;

AJC-18767

The estimation method for unknown thermal properties of ultrathin graphite sheet is suggested in this work. Based on the well known thermal properties of a few materials, the estimation was done by two step approximation algorism. The experimental measurement for four materials of copper, aluminum, indium and graphite was performed at the restricted laboratory environment to get the mutual relation indicating special regularity between those materials. The estimated value of thermal conductivity for a graphite ultrathin thermal sheet was $646 \mathrm{~W} / \mathrm{m} \cdot \mathrm{K}$ that was reasonable comparing to its functional capacity.

1

Keywords: Two step approximation algorism, Ultrathin, Graphite, Thermal conductivity.

\section{INTRODUCTION}

As the modern technology pursues the lighter, smaller, thinner products, the heat dissipating technology becomes more important. The need for faster and broader communication tends core devices to consume more powers, therefore more heat generation. Fast heat spreading and dissipation is a key technology of preventing the core devices from default operation and life-shortening [1,2]. Many kinds of thermal sheets have been used for heat spreading out from core power devices. Indium, aluminum, copper are well known materials for its high thermal performance, but have been suffered from its limited fabricable thickness and cost effectiveness. Graphite has been used in fields of mobile communication and flat panel display industries due to its excellent thermal managing property [3-5]. There have been several kinds of graphite sheets according to its fabrication process and source material treatment. Many graphite sheet providers adduce the proofs of thermal properties such as comparison of thermal flux gradient images and their experimental data. However, the accurate physical values are difficult to get due to complicated measurement instrument and many environmental factors should be considered in application. Some researchers suggested thermal impedance of their products rather than intrinsic physical property values. Sometimes, thermal impedance reflecting many environmental factors during fabrication and application can be more practical and valuable to both of provider and consumer.
In this paper, we suggest a practical method of evaluating thermal properties for the ultrathin graphite sheet that is becoming popular heat spreading part for power system managing. We designed and built the experimental measurement system to get the one dimensional heat spreading data under the vacuum environment to exclude the effect of convection and postulated the two-step approximation algorism. The experiments were performed with four samples of indium, aluminum, copper and graphite to get the positional temperature variation from the heat source. The experimental data of four samples were analyzed with two-step approximation algorism that we built.

Two-step approximation algorism is composed of twostep data fitting processes. The first step is to get the exponential decay fitting formula for four samples of indium, aluminum, cooper and graphite. The empirical data points of positional temperature for four samples could be inspected with experimental equipment. Then secondary fitting process was done for each coefficient to get the extrapolated values about unknown thermal property of ultrathin graphite thermal sheet.

\section{EXPERIMENTAL}

The experimental setup was designed and fabricated as shown in Fig. 1. The temporal positional temperatures were collected by thermistors and recorded by data acquisition unit. The sample was placed inside vacuum chamber to exclude the effect of convection. The chamber was shielded to exclude 

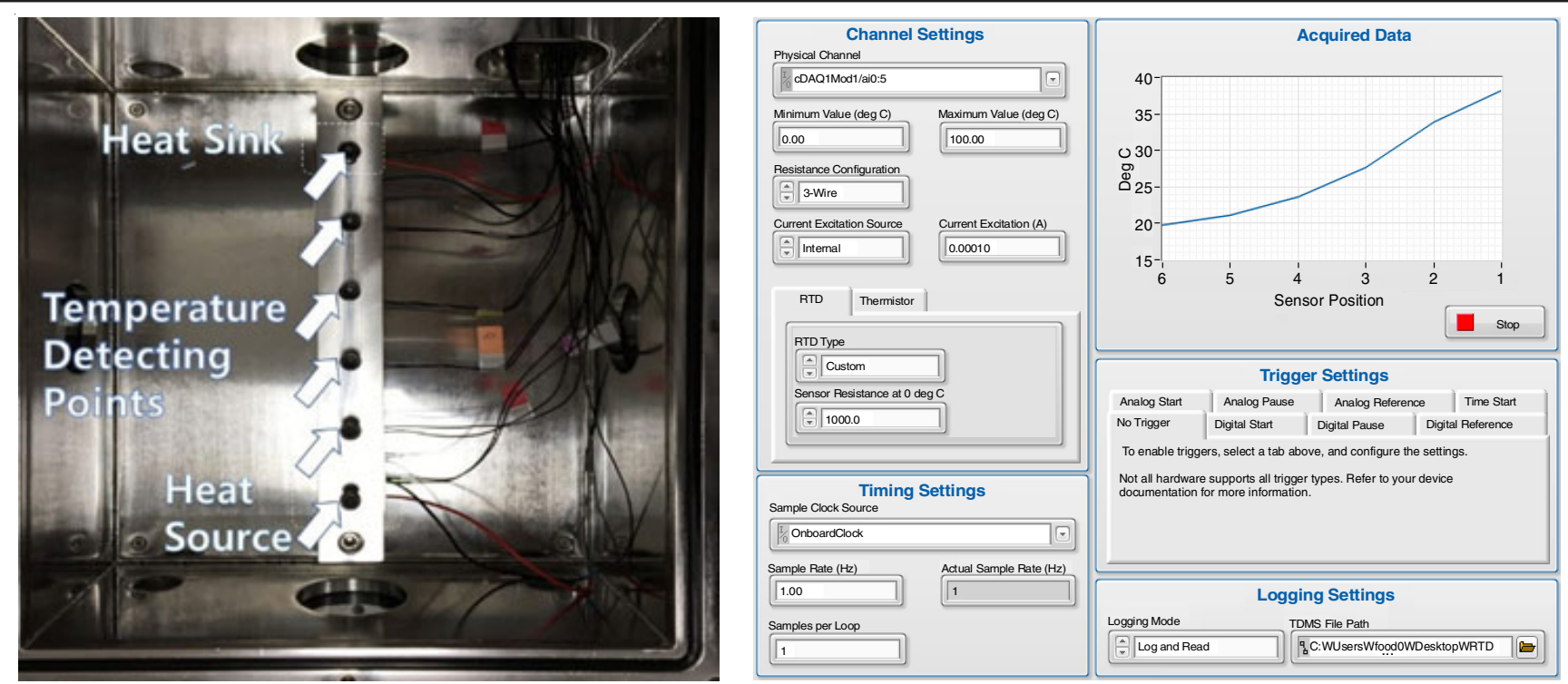

Fig. 1. Measurement instrument (1) vacuum chamber inside of the experimental setup (2) self-designed graphic user interface to monitor the temporal positional temperatures

the effect of environmental radiation. The samples were shaped long to be placed on one dimensional measurement bench. The sample bench was made of Teflon to get a complete contact between the sample and thermistors and isolate any heat spreading out except along the fixed one dimensional bench direction. The heat power biased to the heat source by the electric power control system with providing a certain constant power value.

Six thermistors to detect the positional temperature were placed every $12.5 \mathrm{~mm}$ from the heat source and Teflon was used as the contact surface for accurate temperature measurement. At the end of the one dimensional bench, the heat sink placed to drain the heat from the chamber. The temporal temperature recording was performed and monitored by self-designed graphic user interface as shown in Fig. 1(2). Every samples were prepared with the same shape of length and thickness. The positional temperatures were measured as a function of time. The measurement was done for four materials of indium, aluminum, copper and graphite thermal sheets that have the same thickness under the same environment. After about 20 min the temperatures at every position showed the stable values for every materials. Fig. 2 shows the temperature variation at six positions with time. The thermistors placed every $12.5 \mathrm{~mm}$ from heat source, and the detected temperatures were conveyed to data acquisition board to convert the resistance into temperature. The self-designed graphic user interface displayed the temporal behaviour of thermal variation at every detecting points as a function of distance. In Fig. 2, the first denotes the closest position and the sixth is for the farthest position from the heat source. At every position, the temperatures showed the stable values after $20 \mathrm{~min}$.

\section{RESULTS AND DISCUSSION}

The experiments performed under the same environment for each materials and repeated to reduce the uncertainty. The samples are vacuumed and isolated from outside to exclude any interference factors. In Fig. 2, indium showed the largest gap between the first and second positional temperature and the graphite showed the smallest one. At the position of heat source, indium thermal sheet showed the highest temperature, on the other hand, the graphite thermal sheet showed the lowest one. This phenomenon could be easily explained with their well-known values of thermal conductivity. The reported thermal conductivities of indium, aluminum and copper are about 82, 231 and $401 \mathrm{~W} / \mathrm{m} \cdot \mathrm{K}$, respectively. In case of graphite, thermal conductivity has been reported with many values according to its fabrication process and mass density. The highest one is around $1,800 \mathrm{~W} / \mathrm{m} \cdot \mathrm{K}$ in surface direction, but sometimes showed $15 \mathrm{~W} / \mathrm{m} \cdot \mathrm{K}$ in thickness direction [6,7]. The thermal conductivity, one of important intrinsic physical properties, can be changed by mass density, different fabrication process and application environment. Furthermore, the measurement technology for the thermal conductivity of ultrathin thermal sheet thinner than $1 \mathrm{~mm}$ has not yet officially recognized. Therefore, we propose the empirical two-step approximation method that characterizing thermal properties such as the surface directional thermal conductivity of unknown ultrathin thermal sheet.

From the stable value of positional temperature for four materials, we made an exponential decay fitting to get the fitting coefficients for each thermal sheet as the first step of two-step approximation that we proposed in this paper.

From the fitting formula as shown in Fig. 3, we could find a singularity from three coefficients of the fitting formula. Higher thermal conductivity shows the smaller first and second coefficients and larger coefficient of the right hand side.

Fig. 4 showed the estimated thermal conductivity from the exponential decay fitting formula by the following trial equation:

$$
\mathrm{Y}=\mathrm{y}_{0}+\mathrm{A} \exp ^{-\mathrm{x} / \mathrm{t}}
$$

As the second step of the approximation that we propose in this paper, the calculated thermal conductivities of the graphite sheet are denoted in the graphs with value of 592, 529 and 689. In the experiments, the estimated thermal conductivity consists of two directional properties of the surface directional 

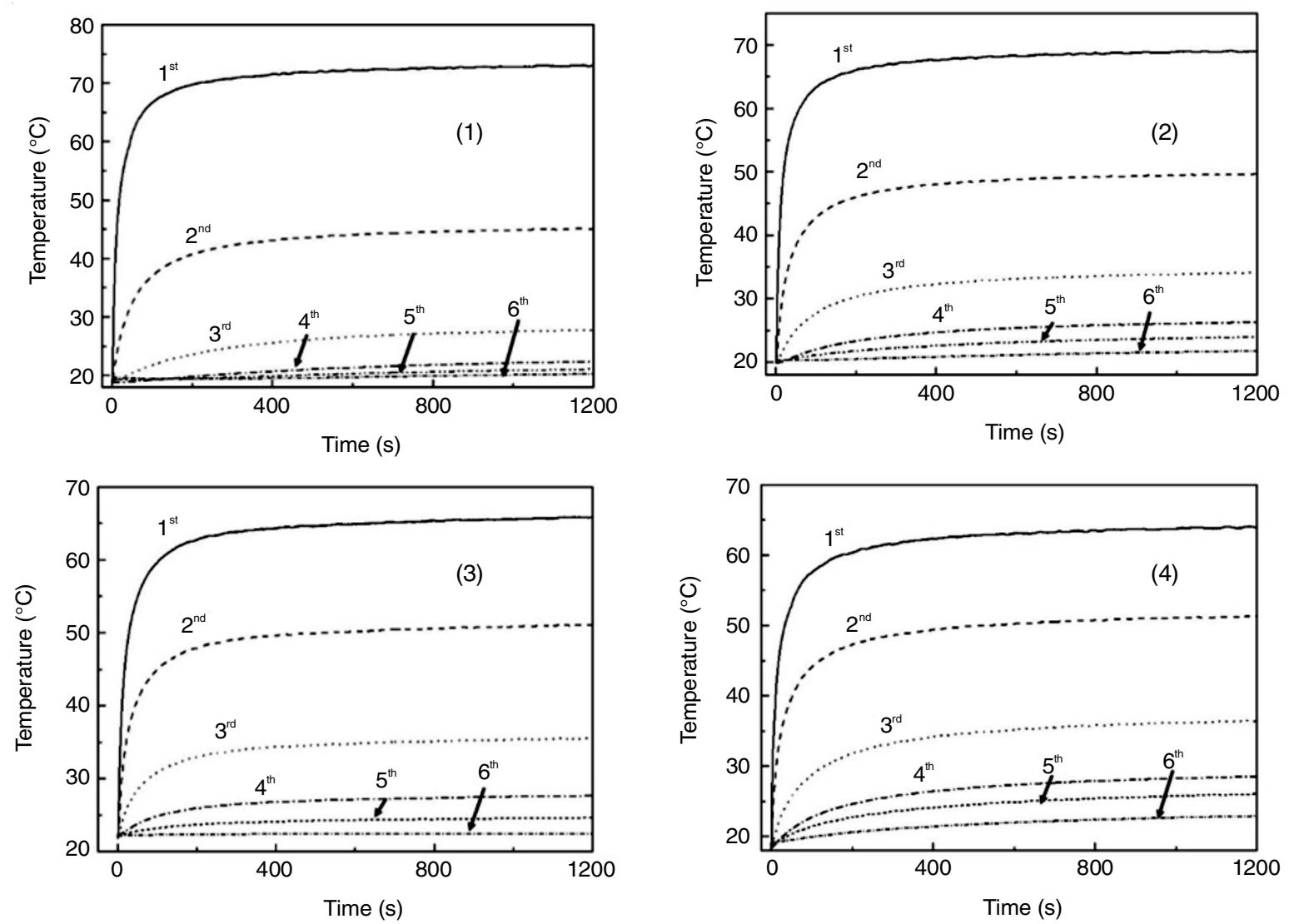

Fig. 2. Positional temperature variation of (1) indium, (2) aluminum, (3) copper and (4) graphite thermal sheet of $100 \mathrm{~mm}$ thickness from heat source
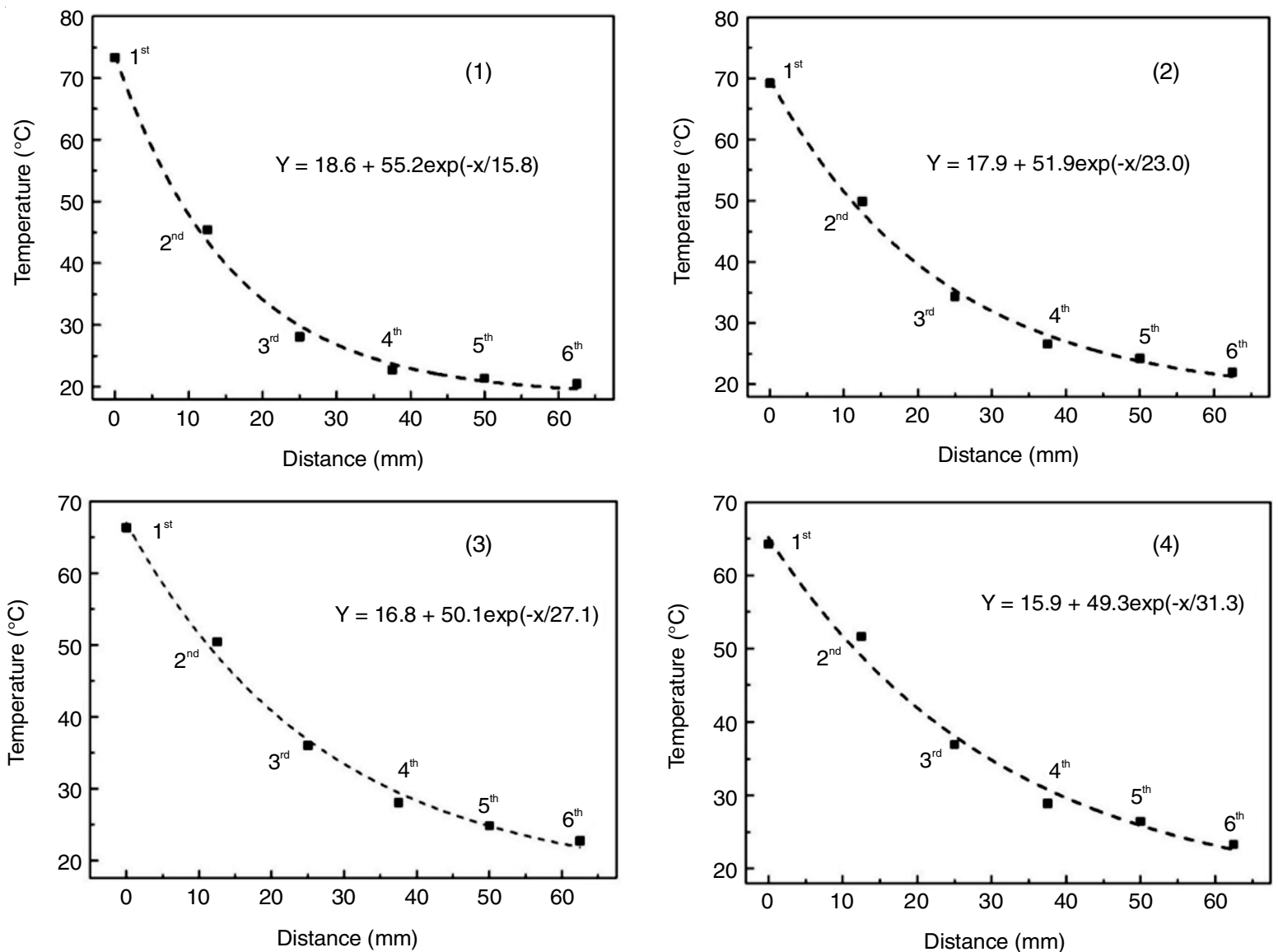

Fig. 3. First step approximation for the stable value of positional temperature (1) indium, (2) aluminum, (3) copper and (4) graphite 

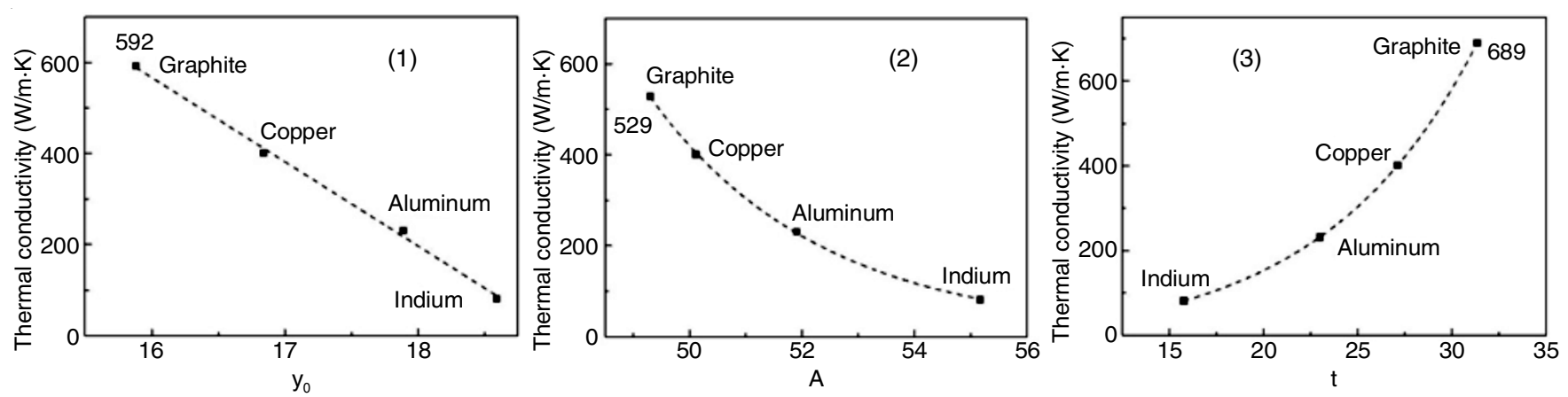

Fig. 4. Estimated thermal conductivity of graphite sheet by exponential decay fitting (1) $y_{0}$, (2) A and (3) t

and the thickness direction. We noticed that these values are in range of commonly recognized thermal property of graphite sheet and the similar values appeared repetitively as shown in Fig. 4. The average value of the estimated thermal conductivity for the graphite sheet that we tested was about $603 \mathrm{~W} /$ $\mathrm{m} \cdot \mathrm{K}$, very close to the reported thermal conductivity of graphite.

\section{Conclusion}

With the fast development of industrial technologies toward the future multiplexed application, the material property under the specific environment can be modified into strange something. Heat dissipation technology has been noticed for long time due to the forthcoming demand for the higher power consumption technology. Many materials have been developed with some creative characteristics and commercialized rapidly. However, their characterization technique could not satisfied the industrial demand due to the complicated measurement equipment and process. In this paper, we proposed the simple two-step approximation method characterizing the thermal property for the unknown material with ultrathin thickness. The ultrathin thermal sheets are provided to many industrial manufacturers concerning heat managing technology in fields of cellular phone fabrication and LED display and lighting. We used the exponential decay approximation to estimate the thermal conductivity of ultrathin graphite sheet, but more systematic and in depth study should be done to reduce the deviation and increase reliability for the values. In this paper, we proposed the empirical two-step approximation method, though not enough, to provide the fast evaluation for the physical property of any unknown material with ultrathin thickness.

\section{ACKNOWLEDGEMENTS}

This research was supported by Space Technology Development Program through the National Research Foundation of Korea (NRF) funded by the Ministry of Education (NRF-2013M1A3A3A02042369).

\section{REFERENCES}

1. E. Monier-Vinard, M.-N. Nguyen, N. Laraqi, V. Bissuel and O. Daniel Steady State Temperature Solution for Early Design of Annealed Pyrolytic Graphite Heat Spreader: Full Results, 15th IEEE ITHERM Conference, p. 945 (2016); https://doi.org/10.1109/ITHERM.2016.7517647.

2. M. Smalc, G. Shives, G. Chen, S. Guggari, J. Norley and R.A. Reynolds III, Thermal Performance of Natural Graphite Heat Spreaders, Proceedings of IPACK 2005, 17-22 July (2005).

3. C.-C. Lee, H.T. Hsu, M.C. Huang and H.H. Huang, Appl. Mech. Mater, 540, 126 (2014); https://doi.org/10.4028/www.scientific.net/AMM.540.126.

4. G.A. Beck, A.A. Carter and J.F. Morris, Measurements of the Thermal Conductivity of Pyrolitic Graphite Substrates for use in SCT Modules, ATL-INDET-98-221 (1998).

5. J.Y. Cho and W.K. Jang, Asian J. Chem., 30, 463 (2018); https://doi.org/10.14233/ajchem.2018.21123.

6. M. Boutinguiza, F. Lusquiños, J. Pou, R. Soto, F. Quintero and R. Comesaña, Opt. Lasers Eng., 50, 727 (2012); https://doi.org/10.1016/j.optlaseng.2011.11.016.

7. X. Zheng, L.W. Wang, R.Z. Wang, T.S. Ge and T.F. Ishugah, Int. J. Heat Mass Transfer, 68, 435 (2014); https://doi.org/10.1016/j.ijheatmasstransfer.2013.09.075. 